\title{
QUALIDAde DE PAINÉIS AGLOMERAdOS HOMOGÊNEOS PRODUZIDOS COM A MADEIRA DE CLONES DE Eucalyptus urophylla
}

Rafael Farinassi Mendes ${ }^{1 *}$, Lourival Marin Mendes ${ }^{1}$, Lilian Lima Mendonça ${ }^{1}$, José Benedito Guimarães Júnior², Fábio Akira Mori ${ }^{1}$

*Autor para correspondência: rafaelfarinassi@gmail.com

RESUMO: O trabalho teve como objetivo avaliar o potencial de clones de Eucalyptus urophylla para produção de painéis aglomerados homogêneos de baixa densidade. Foram avaliados seis clones de Eucalyptus urophylla com 94 meses de idade, plantados no município de Paracatu-MG, sendo utilizada como parâmetro de comparação a madeira de Pinus oocarpa. Para cada clone de Eucalyptus urophylla e também para o Pinus oocarpa foram produzidos seis painéis com o adesivo uréia-formaldeído no teor de $6 \%$, densidade nominal de $0,60 \mathrm{~g} / \mathrm{cm}^{3}$, pressão específica de $40 \mathrm{kgf} / \mathrm{cm}^{2}$, temperatura de $160^{\circ} \mathrm{C}$ e tempo de 8 minutos. Os clones de Eucalyptus urophylla apresentaram potencial para produção de painéis aglomerados convencionais de baixa densidade. Os clones 62 e 28 obtiveram os melhores resultados em todas as propriedades físico-mecânicas avaliadas, apresentando propriedades iguais ou superiores as obtidas para os painéis produzidos com a madeira de Pinus oocarpa. Com relação à norma de comercialização CS 236/1966 apenas o clone 28 atendeu a todos os requisitos. Nenhum dos clones avaliados atendeu a norma EN 312/2003.

Palavras-chave: aglomerados, clones, Eucalyptus urophylla.

\section{QUALITY OF HOMOGENEOUS PARTICLEBOARD PRODUCED WITH Eucalyptus urophylla CLONE WOOD}

\begin{abstract}
The study aimed to evaluate the potential of Eucalyptus urophylla clones to produce homogeneous particleboard of low-density. Six clones of Eucalyptus urophylla were evaluated, at 94 months of age and planted in Paracatu-MG. Pinus oocarpa wood was used as a reference. For each Eucalyptus urophylla clone and also for Pinus oocarpa, six panels were produced with urea-formaldehyde adhesive in the concentration of $6 \%$, nominal density of $0.60 \mathrm{~g} / \mathrm{cm}^{3}$, specific pressure of $40 \mathrm{kgf} / \mathrm{cm}^{2}$, temperature of $160^{\circ} \mathrm{C}$ and a time of 8 minutes. Eucalyptus urophylla clones presented potential for production of conventional particleboard of low density. Clones 62 and 28 showed the best results for all physical and mechanical properties evaluated, presenting properties equal or superior to those obtained for the panels produced with Pinus oocarpa wood. In relation to CS 236/1966 standard, only the clone 28 met all the requirements. None of the clones meets EN 312/2003 standard.
\end{abstract}

Keywords: particleboard, clones, Eucalyptus urophylla.

\section{INTRODUÇÃO}

Atualmente, o setor de painéis de madeira reconstituída tem apresentado grande crescimento no Brasil e no Mundo. No Brasil, isto se deve em função de fatores como a modernização do parque fabril, o surgimento de novos produtos e/ou melhorias de painéis do tipo Oriented Strand Board (OSB), Medium Density Fiberboard (MDF), High Density Fiberboard (HDF), Medium Density Particleboard (MDP) e High Density Particleboard (HDP), além do aumento da demanda dos setores de construção civil e de móveis, os quais são os principais consumidores de painéis de madeira (MENDES, 2011).

1 Universidade Federal de Lavras - Lavras, Minas Gerais, Brasil

2 Universidade Federal do Piauí- Bom Jesus, Piauí, Brasil
As perspectivas são bastante positivas para o setor de painéis de madeira de forma geral, sendo que os segmentos de MDP e MDF os que apresentam um cenário mais positivo. De acordo com dados da Associação Brasileira da Indústria dos Painéis de Madeira - ABIPA (2013), a produção brasileira de painéis aglomerados, no período de 2005 a 2011, evoluiu de 2,048 milhões $\mathrm{m}^{3}$ para 3,069 milhões $\mathrm{m}^{3}$, o que representa um crescimento médio anual de $5,54 \%$.

Com o crescimento da indústria de painéis de madeira, aumenta também a demanda por matéria-prima, o que se torna necessário não apenas o aumento de áreas de plantios com espécies atualmente utilizadas do gênero pinus e eucalipto, mas também a busca de opções

Cerne, Lavras, v. 20, n. 2, p. 329-336, abr./jun. 2014 
de outras espécies de rápido crescimento que possam contribuir de forma quantitativa e qualitativa para suprir as necessidades das indústrias, bem como, contribuir para o desenvolvimento e geração de novos produtos.

Segundo Trianoski (2010) algumas espécies não convencionais, de rápido crescimento, têm apresentado elevado potencial para a produção de painéis de madeira aglomerada. No entanto, por serem oriundas de plantios experimentais ou de pequenas áreas florestais, são ainda incipientes para suprir a necessidade industrial.

Neste sentido, diferentes espécies de eucalipto, que ocupam uma grande área de florestas plantadas, com certo destaque para o estado de Minas Gerais, o qual teve extensas áreas plantadas, inicialmente, visando o suprimento das indústrias siderúrgicas que utilizam carvão vegetal, estão despertando o interesse dos demais setores florestais. Entre as espécies de eucalipto, o Eucalyptus urophylla merece destaque em função das altas taxas de produtividade das florestas plantadas, boa adaptação em grande parte do território brasileiro e disponibilidade de florestas para pronto uso. Sendo assim, o presente trabalho teve o objetivo de avaliar o potencial de clones de Eucalyptus urophylla para produção de painéis aglomerados de baixa densidade.

\section{MATERIAL E MÉTODOS}

\subsection{Matéria-prima}

A madeira de Eucalyptus urophylla foi proveniente de testes clonais instalados no município de Paracatu MG, de propriedade da Companhia Mineira de Metais. Foram selecionados 6 clones com 94 meses de idade, plantados em um espaçamento $10 \times 4 \mathrm{~m}$, em forma de sistema agrossilvopastoril, onde nas entrelinhas foi cultivado arroz no primeiro ano, soja no segundo e pastagem nos anos subseqüentes. Enquanto que a madeira de Pinus oocarpa foi obtida de um plantio experimental aos 8 anos de idade, localizado no campus da Universidade Federal de Lavras (UFLA).

Após a derrubada das árvores de Eucalyptus urophylla e Pinus oocarpa estas foram divididas em toras, sendo também retirados discos para a determinação da densidade básica da madeira de acordo com a norma NBR 11941 (ASSOCIAÇÃO BRASILEIRA DE NORMAS TÉCNICAS - ABNT, 2003) e para a análise química da madeira.

Cerne, Lavras, v. 20, n. 2, p. 329-336, abr./jun. 2014

\subsection{Análise química da madeira}

Para a análise dos constituintes químicos da madeira foi utilizado a serragem obtida pelo processamento da madeira em um moinho Willy. O material utilizado foi aquele que passou pela peneira de 40 mesh e ficou retido na de 60 mesh. Após o material ser acondicionado na temperatura de $20 \pm 2{ }^{\circ} \mathrm{C}$ e umidade relativa de $65 \pm 5 \%$ foram determinados os seus teores de extrativos solúveis em água fria e em água quente (Norma ABTCP(ASSOCIAÇÃO TÉCNICA BRASILEIRA DE CELULOSE E PAPEL ABTCP, 1974) M 4/68), extrativos totais (Norma ABTCP (1974) M 3/69), teor de lignina insolúvel (Norma ABTCP (1974) M 10/71) e holocelulose (obtida pela diferença $\mathrm{H}$ $(\%)=100-\%$ Extrativos totais - \%Teor de lignina).

\subsection{Produção dos painéis aglomerados}

O delineamento experimental se constituiu de sete tratamentos, sendo seis clones de Eucalyptus urophylla (Clones 62, 19, 36, 28, 26 e 58) e um tratamento produzido com a madeira de Pinus oocarpa, o qual foi utilizado como parâmetro de comparação. Para cada um dos tratamentos foram produzidos seis painéis homogêneos, com densidade nominal de $0,60 \mathrm{~g} / \mathrm{cm}^{3}$ e dimensões de $480 \times 480 \times 15$ $\mathrm{mm}$ (comprimento, largura e espessura, respectivamente).

As toras foram desdobradas em uma serra de fita, dando origem a tábuas com aproximadamente $20 \mathrm{~mm}$ de espessura, sendo posteriormente processadas em uma serra circular, originando blocos de madeira, os quais, após serem picados manualmente com facão, passaram por um moinho martelo com peneira de abertura de $6,14 \mathrm{~mm}$, obtendo assim as partículas do tipo sliver.

As partículas obtidas foram secas a uma umidade de 3\% (base massa seca das partículas) e levadas para uma encoladeira do tipo tambor giratório para a aplicação de $6 \%$ de adesivo uréia-formaldeído (Sólidos de adesivo em relação a massa seca de partícula). As partículas encoladas foram então depositadas em uma caixa formadora com dimensões laterais de $48 \times 48 \mathrm{~cm}$ (comprimento x largura), onde sofreu uma pré-prensagem a frio a $8 \mathrm{kgf} / \mathrm{cm}^{2}$, sendo o colchão de partículas obtido conduzido para prensagem a quente com temperatura de $160^{\circ} \mathrm{C}$, pressão específica de $40 \mathrm{kgf} / \mathrm{cm}^{2}$ e por período de 8 minutos. 


\subsection{Determinação das propriedades dos painéis e análise estatística}

Os corpos de prova foram confeccionados dos painéis após climatizados na temperatura de $20 \pm 2^{\circ} \mathrm{C}$ e umidade relativa de $65 \pm 5 \%$. Na avaliação das propriedades absorção de água, inchamento em espessura, ligação interna e compressão paralela a superfície do painel foi empregada a norma ASTM - D1037 (AMERICAN SOCIETY FOR TESTING AND MATERIALS - ASTM, 2006) e para as propriedades módulo de ruptura e módulo de elasticidade na flexão estática foi empregada a norma DIN 52362 (NORMEN FÜR HOLZFASERPLATEN SPANPLATTEN SPERRHOLZ - DIN, 1982).

A análise estatística foi conduzida em delineamento inteiramente casualizado. $\mathrm{O}$ teste de média de Tukey, a 5\% de significância, foi utilizado para a comparação das propriedades físico-mecânicas dos painéis entre os clones de Eucalyptus urophylla. Enquanto que o teste de Dunnett, também a 5\% de significância, foi utilizado para comparar as propriedades dos painéis aglomerados obtidas para cada clone de Eucalyptus urophylla com os valores de cada propriedade obtido pelos painéis de Pinus oocarpa (Parâmetro de comparação).

\section{RESULTADOS E DISCUSSÃO}

\subsection{Análise química e densidade básica da madeira e densidade aparente dos painéis}

Os valores médios obtidos para os constituintes químicos da madeira dos clones de Eucalyptus urophylla e de Pinus oocarpa estão apresentados na Tabela 1.

Os valores médios de extrativos totais, lignina e holocelulose variaram na faixa de 6,35 a $8,91 \% ; 22,76$ a $29,21 \%$ e de 62,14 a $70,89 \%$, respectivamente. Enquanto que para a madeira de Pinus oocarpa os valores médios foram de $6,83 \%, 26,67 \%$ e $66,50 \%$, respectivamente.

Trugilho et al. (1996) avaliando clones de Eucalyptus saligna obtiveram valores de extrativos totais entre 3,68 a $5,24 \%$, de lignina entre 24,32 a $27,04 \%$ e de holocelulose entre 68,52 a 72,48\%. Enquanto Queiroz et al. (2004) avaliando a madeira do hibrido de Eucalyptus grandis x Eucalyptus urophylla obteve valores médios de 26,7 e $27,2 \%$ para lignina e de 3,45 e $2,73 \%$ para os extrativos, para híbridos com densidades de 0,55 e 0,45 $\mathrm{g} / \mathrm{cm}^{3}$, respectivamente. De forma geral, observa-se que
Tabela 1 - Análise química da madeira.

Table 1 -Wood chemical analysis.

\begin{tabular}{cccc}
\hline Espécie & Extrativos (\%) & Lignina (\%) & Holocelulose (\%) \\
\hline Clone 62 & 6,35 & 22,76 & 70,89 \\
Clone 19 & 8,81 & 26,19 & 65,00 \\
Clone 36 & 8,91 & 26,72 & 64,37 \\
Clone 28 & 6,91 & 24,12 & 68,97 \\
Clone 26 & 8,65 & 29,21 & 62,14 \\
Clone 58 & 8,52 & 26,47 & 65,01 \\
P. oocarpa & 6,83 & 26,67 & 66,50 \\
\hline
\end{tabular}

o valor de lignina e holocelulose foram próximos dos valores encontrados em literatura. Os valores de extrativos se mostraram um pouco acima dos obtidos em literatura.

Morais et al. (2005) analisando a composição química da madeira do Pinus oocarpa com oito anos de idade obtiveram valores médios de $80,27 \%$ de holocelulose, $25,18 \%$ de lignina e $9,95 \%$ de extrativos totais. De forma geral, observa-se maior valor de holocelulose e menores valores de extrativos que os obtidos em literatura para a madeira de Pinus oocarpa. Os valores encontrados para lignina se mostraram coerentes com os encontrados em literatura.

Os valores médios obtidos para a densidade básica da madeira dos clones de Eucalyptus urophylla e de Pinus oocarpa, assim como a densidade aparente dos painéis e suas respectivas razão de compactação estão apresentados na Tabela 2.

$\mathrm{Na}$ análise da densidade básica da madeira, os valores médios variaram na faixa de 0,52 a $0,65 \mathrm{~g} / \mathrm{cm}^{3}$

Tabela 2 - Densidade básica da madeira e densidade aparente e razão de compactação dos painéis (RC).

Table 2 - Wood basic density and bulk density and compaction ratio of the panels $(R C)$.

\begin{tabular}{cccc}
\hline Espécie & $\begin{array}{c}\text { Densidade básica da } \\
\text { madeira }\left(\mathrm{g} / \mathrm{cm}^{3}\right)\end{array}$ & $\begin{array}{c}\text { Densidade aparente } \\
\text { dos painéis }\left(\mathrm{g} / \mathrm{cm}^{3}\right)\end{array}$ & RC \\
\hline Clone 62 & 0,53 & 0,55 & 1,04 \\
Clone 19 & 0,65 & 0,55 & 0,85 \\
Clone 36 & 0,59 & 0,58 & 0,98 \\
Clone 28 & 0,52 & 0,54 & 1,04 \\
Clone 26 & 0,60 & 0,54 & 0,90 \\
Clone 58 & 0,56 & 0,56 & 1,00 \\
P. oocarpa & 0,50 & 0,55 & 1,10 \\
\hline
\end{tabular}

Cerne, Lavras, v. 20, n. 2, p. 329-336, abr./jun. 2014 
para os clones de Eucalyptus urophylla, enquanto que para a madeira de Pinus oocarpa o valor médio obtido foi de $0,50 \mathrm{~g} / \mathrm{cm}^{3}$. Queiroz et al. (2004) avaliando a madeira do hibrido de Eucalyptus grandis $\mathrm{x}$ Eucalyptus urophylla encontraram valores médios para dois híbridos de seis anos de idade de 0,55 e $0,45 \mathrm{~g} / \mathrm{cm}^{3}$.

Os valores médios da densidade aparente dos painéis variaram na faixa de $0,54 \mathrm{a} 0,58 \mathrm{~g} / \mathrm{cm}^{3}$ para os clones e teve o valor médio de $0,55 \mathrm{~g} / \mathrm{cm}^{3}$ para os painéis de Pinus oocarpa. Sendo, portanto os painéis classificados como de baixa densidade de acordo com Iwakiri (2005). As densidades aparentes médias dos painéis também se diferiram da densidade nominal estipulada de $0,60 \mathrm{~g} / \mathrm{cm}^{3}$. Fato que pode ser justificado devido à perda de partículas durante a formação do colchão, assim como também pode estar relacionado ao retorno em espessura dos painéis após a retirada da prensa quente e acondicionamento, com conseqüente aumento do volume dos painéis e redução da densidade nominal inicial.

Em relação a razão de compactação, nenhum dos painéis produzidos apresentaram valores médios estipulados como ideais por Maloney (1993) e Tsoumis (1991), que é na faixa de 1,3 a 1,6. Em alguns casos a razão de compactação foi inferior a 1,0; o que implica que não houve densificação adequada do material.

\subsection{Propriedades físicas}

\subsubsection{Absorção de água}

Os valores médios obtidos para as propriedades de absorção de água após duas e vinte e quatro horas de imersão (AA2h e AA24h) estão apresentados na Tabela 3.

De acordo com a análise dos dados, foi observada diferença significativa entre os clones de eucalipto para AA2h, sendo os clones 62 e 28 os que apresentaram o melhor desempenho, porém estatisticamente iguais aos outros tratamentos, com exceção do clone 26 , o qual apresentou o maior valor médio de absorção de água. Tal fato pode estar relacionado, em parte, com a menor razão de compactação dos painéis desse tratamento (Tabela 2) em comparação com a maioria dos tratamentos, o que faz com que permita uma maior facilidade de entrada de água no painel. Não foi observada relação dessa propriedade com a análise química da madeira (Tabela 1).

Ainda para a propriedade de AA2h, quando realizado a comparação entre os painéis produzidos com a madeira de clones de eucalipto e aqueles com a madeira de
Tabela 3 - Valores médios de absorção de água.

Table 3 - Average values of water absorption.

\begin{tabular}{ccccccc}
\hline Espécie & \multicolumn{2}{c}{$\begin{array}{c}\text { AA2h } \\
(\%)\end{array}$} & $\begin{array}{c}\text { Teste } \\
\text { Dunnett }\end{array}$ & $\begin{array}{c}\text { AA24h } \\
(\%)\end{array}$ & $\begin{array}{c}\text { Teste } \\
\text { Dunnett }\end{array}$ \\
\hline Clone 62 & 37,9 & AB & $0,0001^{*}$ & 104,7 & A & $0,3132^{\mathrm{ns}}$ \\
Clone 19 & 51,9 & BC & $0,0229^{*}$ & 94,1 & A & $0,0143^{*}$ \\
Clone 36 & 56,0 & BC & $0,0721^{\mathrm{ns}}$ & 103,8 & A & $0,2554^{\mathrm{ns}}$ \\
Clone 28 & 35,7 & AB & $0,0002^{*}$ & 107,4 & A & $0,5914^{\mathrm{ns}}$ \\
Clone 26 & 63,9 & C & $0,4153^{\mathrm{ns}}$ & 108,8 & A & $0,6763^{\mathrm{ns}}$ \\
Clone 58 & 52,0 & BC & $0,0240^{*}$ & 98,7 & A & $0,0649^{\mathrm{ns}}$ \\
P. oocarpa & 77,4 & & & 117,8 & & \\
\hline
\end{tabular}

Médias seguidas de mesma letra maiúscula na coluna, não diferem entre si pelo teste Tukey com um nível nominal de significância de 5\%. * Difere estatisticamente pelo teste de dunnett, a 5\% de significância, dos painéis produzidos com Pinus oocarpa (Parâmetro de comparação). ns não- significativo pelo teste de dunnett a $5 \%$ de significância.

Pinus oocarpa, foi possível verificar pelo teste de Dunnett, que os painéis produzidos com pinus não apresentaram diferença significativa em relação apenas aos clones 36 e 26, obtendo em relação aos outros clones maior valor médio de AA2h.

Para a propriedade de AA24h, não foi observado diferença estatística entre os clones, porém quando comparados, pelo teste de dunnett, com os painéis de pinus, o clone 19 apresentou diferença significativa, obtendo valor médio de absorção de água menor. $\mathrm{O}$ fato pode estar relacionado com a associação de dois fatores, sendo estes: 1) A constituição química da madeira, principalmente no teor de extrativos, onde a madeira do clone 19 apresentou valor médio superior $(8,81 \%)$ em relação a madeira de Pinus oocarpa (6,83\%); e 2) a menor razão de compactação dos painéis do Clone $19(0,85)$ em relação aos painéis de pinus $(1,10)$, o que diferentemente do caso da AA2h, que uma maior razão de compactação promove uma barreira a penetração de água, no período de AA24h já houve tempo suficiente para total penetração de água no painel, sendo mais influenciada pelo número de partículas com grupos hidroxílicos para ligação com moléculas de água, o que ocorre nos painéis com maior razão de compactação.

Em estudo realizado por Naumann et al. (2008) avaliando a mistura da madeira de Eucalyptus urophylla com a madeira de paricá, foi observado para os painéis produzidos apenas com a madeira de eucalipto, com

Cerne, Lavras, v. 20, n. 2, p. 329-336, abr./jun. 2014 
densidade nominal de $0,60 \mathrm{~g} / \mathrm{cm}^{3}$ e $8 \%$ de teor de adesivo uréia-formaldeído, valores médios de 87,8 e 101,9\% para as propriedades de AA2h e AA24h, respectivamente. Enquanto que Del Menezzi et al. (1996) estudando a mistura das espécies Eucalyptus urophylla e Pinus oocarpa para a produção de painéis aglomerados com densidade nominal de $0,7 \mathrm{~g} / \mathrm{cm}^{3}$ e $10 \%$ de teor de adesivo uréia-formaldeído, obtiveram valores médios na faixa de 21,3 a $74,5 \%$ e de 62,2 a $87,0 \%$ para as propriedades de AA2h e AA24h, respectivamente. Neste sentido, o comportamento dos painéis produzidos com os diferentes clones de eucalipto se mostrou de acordo com os resultados encontrados na literatura.

\subsubsection{Inchamento em espessura e taxa de não-retorno em espessura}

Os valores médios obtidos para as propriedades de inchamento em espessura após duas e vinte e quatro horas de imersão em água (IE2h e IE24h) e para a taxa de não-retorno em espessura (TNRE) estão apresentados na Tabela 4.

Para a propriedade de IE2h, os clones 28 e 62 foram os que apresentaram o melhor desempenho, porém sendo estatisticamente iguais aos clones 36 e 19, contudo diferente estatisticamente dos clones $26 \mathrm{e} 58$, os quais obtiveram maior valores médios. O que também pode estar

Tabela 4 - Valores médios de inchamento em espessura e taxa de não-retorno em espessura.

Table 4 - Average values of thickness swelling and thickness non-return rate.

\begin{tabular}{|c|c|c|c|c|c|c|}
\hline Espécie & $\begin{array}{c}\text { IE } 2 \mathrm{~h} \\
(\%)\end{array}$ & $\begin{array}{c}\text { Teste } \\
\text { Dunnett }\end{array}$ & $\begin{array}{l}\text { IE } 24 \mathrm{~h} \\
(\%)\end{array}$ & $\begin{array}{c}\text { Teste } \\
\text { Dunnett }\end{array}$ & $\begin{array}{l}\text { TNRE } \\
(\%)\end{array}$ & $\begin{array}{c}\text { Teste } \\
\text { Dunnett }\end{array}$ \\
\hline Clone 62 & $13,8 \mathrm{AB}$ & 0,0071 * & $31,2 \mathrm{~A}$ & $0,9999 \mathrm{~ns}$ & $29,2 \mathrm{~A}$ & $0,0722^{\mathrm{ns}}$ \\
\hline Clone 19 & $16,7 \mathrm{BC}$ & $0,1695^{\text {ns }}$ & 29,9 A & $0,8226^{\mathrm{ns}}$ & $25,6 \mathrm{~A}$ & $0,9992^{\mathrm{ns}}$ \\
\hline Clone 36 & $20,5 \mathrm{BC}$ & $0,9933^{\mathrm{ns}}$ & 29,9 A & $0,8013^{\mathrm{ns}}$ & $26,7 \mathrm{~A}$ & $0,8210^{\mathrm{ns}}$ \\
\hline Clone 28 & $13,3 \mathrm{AB}$ & $0,0075^{*}$ & 29,9 A & $0,8534^{\mathrm{ns}}$ & $27,3 \mathrm{~A}$ & $0,6312^{\mathrm{ns}}$ \\
\hline Clone 26 & $28,8 \quad \mathrm{D}$ & 0,0183 * & 40,8 B & 0,0001 * & 36,3 B & 0,0001 * \\
\hline Clone 58 & $22,0 \mathrm{CD}$ & 0,9999 ns & $32,7 \mathrm{~A}$ & $0,9351^{\mathrm{ns}}$ & $29,4 \mathrm{~A}$ & $0,0593^{\mathrm{ns}}$ \\
\hline P. oocarpa & 21,6 & & 31,5 & & 25,1 & \\
\hline
\end{tabular}

Médias seguidas de mesma letra maiúscula na coluna, não diferem entre si pelo teste Tukey com um nível nominal de significância de 5\%. * Difere estatisticamente pelo teste de dunnett, a 5\% de significância, dos painéis produzidos com Pinus oocarpa (Parâmetro de comparação). ${ }^{\text {ns }}$ não- significativo pelo teste de dunnett a 5\% de significância. associado, assim como explicado na propriedade de AA2h, ao fato da maior razão de compactação desses painéis, o que inicialmente promove uma barreira de entrada à água.

Ainda em relação a propriedade de IE2h, apenas os clones 28, 62 e 26 apresentaram, pelo teste de dunnett, diferença significativa em relação aos painéis produzidos com a madeira de Pinus oocarpa, sendo os dois primeiros (clones 28 e 62) com valores médios menores em relação ao parâmetro de comparação (Pinus oocarpa) e o clone 26 com maior valor médio.

$\mathrm{Na}$ análise das propriedades de IE24h e TNRE, apenas o clone 26 diferenciou-se dos demais clones de eucalipto, sendo obtido para este clone os maiores valores médios. Em relação a madeira de Pinus oocarpa, foi obtida pelo teste de dunnett, igualdade estatística com todos os clones de eucalipto, com exceção do clone 26, o qual obteve maior valor médio para estas propriedades. Não foi observado efeito claro da constituição química (Tabela 1) e da razão de compactação (Tabela 2) sobre esse resultado, podendo então estar associado as características anatômicas do clone 26.

Ainda em questão da TNRE, observa-se que ela tem relação direta com a propriedade IE24h, sendo que quanto maior o IE24h maior a TNRE. O fato é devido à maior variação em espessura resultante da liberação das tensões de compressão, o qual se torna parcialmente irrecuperável.

Iwakiri et al. (2000), avaliando a produção de painéis aglomerados com densidade nominal de $0,75 \mathrm{~g} /$ $\mathrm{cm}^{3}$, teores de 8 e $12 \%$ do adesivo uréia-formaldeído e três espécies de eucaliptos (Eucalyptus maculata, Eucalyptus grandis e Eucalyptus tereticornis) obtiveram valores médios nas faixas de 12,4 a $30,6 \%$ e de 23,5 a $38,8 \%$ para as propriedades de IE2h e IE24h, respectivamente.

Neste sentido, os valores médios obtidos para os seis clones de Eucalyptus urophylla se mostraram satisfatórios com os encontrados em literatura. No entanto, em comparação com as normas de comercialização, os valores médios obtidos para o IE24h em todos os tratamentos não atenderam a norma EN 312 (EUROPEAN COMMITTEE FOR STANDARDIZATION - EN, 2003), que estipula o valor máximo de $15 \%$ (Tipo 4). Entretanto, os clones 19, 36 e 28 conseguiram atender a a norma Commercial Standard- CS 236-66 (COMMERCIAL STANDARD - CS, 1968), que estipula valores máximos de inchamento em espessura após vinte e quatro horas de imersão de $35 \%$.

Cerne, Lavras, v. 20, n. 2, p. 329-336, abr./jun. 2014 


\subsection{Propriedades mecânicas}

\subsubsection{Módulo de elasticidade (MOE) e Módulo de ruptura (MOR) à flexão estática}

Os valores médios obtidos para as propriedades de módulo de elasticidade (MOE) e módulo de ruptura (MOR) à flexão estática estão apresentados na Tabela5.

Tabela 5 - Valores médios de módulo de elasticidade e módulo de ruptura.

Table 5 - Average values of the modulus of elasticity and modulus of rupture.

\begin{tabular}{ccccccc}
\hline Espécie & \multicolumn{2}{c}{ MOE $(\mathrm{MPa})$} & $\begin{array}{c}\text { Teste } \\
\text { Dunnett }\end{array}$ & MOR $(\mathrm{MPa})$ & $\begin{array}{c}\text { Teste } \\
\text { Dunnett }\end{array}$ \\
\hline Clone 62 & 1284,9 & $\mathrm{~A}$ & $0,2758 \mathrm{~ns}$ & 5,5 & $\mathrm{AB}$ & $0,3900 \mathrm{~ns}$ \\
Clone 19 & 975,6 & $\mathrm{BC}$ & $0,0555 \mathrm{~ns}$ & 3,1 & $\mathrm{C}$ & $0,0540 \mathrm{~ns}$ \\
Clone 36 & 1143,0 & $\mathrm{AB}$ & $0,9999 \mathrm{~ns}$ & 4,8 & $\mathrm{AB}$ & $0,9990 \mathrm{~ns}$ \\
clone 28 & 1357,3 & $\mathrm{~A}$ & $0,0303^{*}$ & 6,2 & $\mathrm{~A}$ & $0,0395^{*}$ \\
clone 26 & 927,3 & $\mathrm{C}$ & $0,0098^{*}$ & 3,0 & $\mathrm{C}$ & $0,0425^{*}$ \\
clone 58 & 1043,5 & $\mathrm{BC}$ & $0,3783 \mathrm{~ns}$ & 3,8 & $\mathrm{BC}$ & $0,5211 \mathrm{~ns}$ \\
Pinus oocarpa & 1157,6 & & & 4,6 & & \\
\hline
\end{tabular}

Médias seguidas de mesma letra maiúscula na coluna, não diferem entre si pelo teste Tukey com um nível nominal de significância de 5\%. * Difere estatisticamente pelo teste de dunnett, a $5 \%$ de significância, dos painéis produzidos com Pinus oocarpa (Parâmetro de comparação). ns não- significativo pelo teste de dunnett a 5\% de significância.

Para ambas as propriedades, os clones 62, $36 \mathrm{e}$ 28 apresentaram o melhor desempenho, sendo iguais estatisticamente. $\mathrm{O}$ que em parte pode estar associado aos maiores valores médios de razão de compactação desses painéis (Tabela 2), assim como também com as características de resistência de cada tipo de madeira. Não foi observado efeito claro da química da madeira (Tabela 1) sobre os valores de MOE e MOR à flexão estática.

Enquanto que em comparação dos clones de eucalipto com os painéis de Pinus oocarpa, houve diferença significativa apenas para os clones 28 e 26 , sendo que o clone 28 obteve os maiores valores médios, o que pode estar associado a resistência mecânica proporcionado pelo tipo de madeira, visto que em relação a razão de compactação os painéis de pinus apresentaram valor médio maior. O clone 26 obteve os menores valores médios em relação ao parâmetro de comparação para as duas propriedades. Não foi observado efeito claro da constituição química (Tabela 1) e da razão de compactação (Tabela 2) sobre esse resultado.

Guimarães Júnior (2008) avaliando a produção de painéis aglomerados de média densidade, com o adesivo uréia-formaldeído em um teor de $8 \%$ e diferentes procedências de madeira das espécies Eucalyptus cloeziana, Eucayptus grandis e Eucalyptus saligna, obteve valores médios para o MOE na faixa de 668,4 a 10555,9 MPa. Enquanto que Del Menezzi et al. (1996) estudando a produção de painéis aglomerados com a mistura da madeira de Pinus elliottii e Eucalyptus urophylla, densidade nominal dos painéis de $0,70 \mathrm{~g} / \mathrm{cm}^{3}$ e $10 \%$ de teor do adesivo uréia-formaldeído obtiveram valor médio para MOR de 8,7 MPa para os painéis produzidos apenas com Eucalyptus urophylla.

Neste contexto, quando comparado os valores médios obtidos para os clones com os valores encontrados em literatura, todos os clones apresentaram resultados satisfatórios para o MOE e inferiores aos obtidos para o MOR. Em comparação com as normas de comercialização, os valores médios obtidos para MOE e MOR não atenderam a norma EN 312 (EN, 2003), que estipula os valores minimos de 1600 e $13 \mathrm{MPa}$, respectivamente. Entretanto, os clones 62,36 e 28 conseguiram atender a norma CS 236-66 (CS, 1968) para o MOE, a qual determina o valor mínimo de 1052,1 MPa. Em relação ao MOR apenas o clone 28 conseguiu atender o mínimo estipulado de 5,6 MPa pela norma CS 236-66 (CS, 1968).

\subsubsection{Compressão paralela e ligação interna}

Os valores médios obtidos para as propriedades de compressão paralela e ligação interna estão apresentados na Tabela 6.

$\mathrm{Na}$ análise da propriedade compressão paralela foi observada igualdade estatística entre os clones, com exceção do clone 26 que apresentou diferença significativa em relação aos clones 62 e 28 , os quais, por sua vez, obtiveram os maiores valores médios. Na comparação dos painéis produzidos com os clones de eucalipto com os painéis produzidos com a madeira de Pinus oocarpa, foi observada diferença significativa apenas para o clone 26 , sendo obtido para este clone valor médio inferior aos painéis de Pinus oocarpa.

Para a propriedade ligação interna, os clones 62 , 36 e 28 apresentaram o melhor desempenho, sendo iguais estatisticamente. Enquanto que em comparação dos clones de eucalipto com os painéis de Pinus oocarpa, houve diferença significativa apenas para os clones 19 e 26, sendo que ambos apresentaram menores valores médios

Cerne, Lavras, v. 20, n. 2, p. 329-336, abr./jun. 2014 
em relação aos painéis de Pinus oocarpa. Os clones 19 e 26 foram os que apresentaram os menores valores de razão de compactação (Tabela 2), comprovando assim a relação direta desse parâmetro com a propriedade ligação interna.

Tabela 6 - Valores médios de compressão paralela e ligação interna.

Table 6 - Average values of parallel compression and internal bond.

\begin{tabular}{ccccccc}
\hline Espécie & $\begin{array}{c}\text { Compressão } \\
(\mathrm{MPa})\end{array}$ & $\begin{array}{c}\text { Teste } \\
\text { Dunnett }\end{array}$ & $\begin{array}{c}\text { Ligação interna } \\
(\mathrm{MPa})\end{array}$ & $\begin{array}{c}\text { Teste } \\
\text { Dunnett }\end{array}$ \\
\hline clone 62 & 4,3 & $\mathrm{~A}$ & $0,6637 \mathrm{~ns}$ & 0,22 & $\mathrm{AB}$ & $0,9991 \mathrm{~ns}$ \\
clone 19 & 3,0 & $\mathrm{AB}$ & $0,3609 \mathrm{~ns}$ & 0,17 & $\mathrm{BC}$ & $0,0169 *$ \\
clone 36 & 3,1 & $\mathrm{AB}$ & $0,4793 \mathrm{~ns}$ & 0,22 & $\mathrm{AB}$ & $0,9999 \mathrm{~ns}$ \\
clone 28 & 4,4 & $\mathrm{~A}$ & $0,5794 \mathrm{~ns}$ & 0,26 & $\mathrm{~A}$ & $0,1027 \mathrm{~ns}$ \\
clone 26 & 2,3 & $\mathrm{~B}$ & $0,0076^{*}$ & 0,14 & $\mathrm{C}$ & $<0,0001^{*}$ \\
clone 58 & 3,2 & $\mathrm{AB}$ & $0,6466 \mathrm{~ns}$ & 0,19 & $\mathrm{~B}$ & $0,2409 \mathrm{~ns}$ \\
Pinus oocarpa & 3,8 & & & 0,22 & & \\
\hline
\end{tabular}

Médias seguidas de mesma letra maiúscula na coluna, não diferem entre si pelo teste Tukey com um nível nominal de significância de 5\%. * Difere estatisticamente pelo teste de dunnett, a 5\% de significância, dos painéis produzidos com Pinus oocarpa (Parâmetro de comparação). ns não- significativo pelo teste de dunnett a $5 \%$ de significância.

Guimarães Júnior (2008) avaliando a produção de painéis aglomerados de média densidade, com o adesivo uréia-formaldeído em um teor de $8 \%$ e diferentes procedências de madeira das espécies Eucalyptus cloeziana, Eucayptus grandis e Eucalyptus saligna, obteve valores médios para a propriedade compressão paralela na faixa de 4,5 a 6,7 MPa.

Pedrazzi et al. (2006) verificando a produção de painéis aglomerados com o resíduo gerado pela indústria de celulose após o processamento da madeira de Eucalyptus saligna, obtiveram valores médios de ligação interna na faixa de 0,14 a $0,18 \mathrm{MPa}$ para os painéis produzidos com a utilização de $8 \%$ de adesivo uréia-formaldeído e densidade do painel de $0,65 \mathrm{~g} / \mathrm{cm}^{3}$

Neste contexto, para a propriedade compressão paralela, os valores obtidos nesse estudo ficaram abaixo dos encontrados em literatura. Enquanto que para a propriedade ligação interna, os valores se mostraram adequados quando comparados com os valores encontrados em literatura. Em comparação com as normas de comercialização, apenas os painéis produzidos com o clone 28 atendeu ao minimo requerido pela norma EN 312 (EN, 2003) de 0,24 MPa para propriedade de ligação interna. Entretanto, em comparação com a norma CS 236-66 (CS, 1968), a qual determina o valor mínimo de $0,14 \mathrm{MPa}$, todos os tratamentos atenderam ao mínimo requerido.

\section{CONCLUSÕES}

De forma geral, os clones de Eucalyptus urophylla apresentam-se com potencial para produção de painéis aglomerados convencional de baixa densidade.

Os clones 62 e 28 obtiveram os melhores resultados em todas as propriedades físico-mecânicas avaliadas. Apesar de que em relação à norma de comercialização CS 236 (CS, 1966) apenas o clone 28 atendeu a todos os requisitos. Nenhum dos clones avaliados atendeu a norma EN 312 (EN, 2003).

Em comparação com os painéis produzidos com a madeira de Pinus oocarpa, os clones 62, 36, 58 e 28 se apresentaram com propriedades físico-mecânicas iguais ou superiores.

O clone 26 foi o que apresentou os piores resultados para as propriedades físico-mecânicas.

Os valores médios obtidos para MOE e MOR não atenderam a norma EN 312 (EN, 2003), que estipula os valores minimos de 1600 e $13 \mathrm{MPa}$, respectivamente.

\section{REFERÊNCIAS}

AMERICAN SOCIETY FOR TESTING AND MATERIALS. Annual book of ASTM: D-1037: standard methods of evaluating properties of wood-base fiber and particles materials. Philladelphia, 2006.

ASSOCIAÇÃO BRASILEIRA DA INDÚSTRIA DOS PAINÉIS DE MADEIRA. Números. Disponível em: $<$ http://www.abipa.org.br/numeros.php $>$. Acesso em: 10 fev. 2013.

ASSOCIAÇÃO BRASILEIRA DE NORMAS

TÉCNICAS. NBR 11941: madeira, determinação da densidade básica. Rio de Janeiro, 2003. 6 p.

ASSOCIAÇÃO TÉCNICA BRASILEIRA DE CELULOSE E PAPEL. Normas técnicas. São Paulo, 1974.

COMMERCIAL STANDARD. CS 236-66: mat formed wood particleboard. Geneva, 1968.

DEL MENEZZI, C. H. S.; SOUZA, M. R.; GONÇALEZ, J. C. Fabricação e avaliação tecnológica

Cerne, Lavras, v. 20, n. 2, p. 329-336, abr./jun. 2014 
da chapa aglomerada de mistura de Eucalyptus urophylla T. S. Blake e Pinus oocarpa Schiede. Revista Árvore, Viçosa, MG, v. 20, n. 3, p. 371-379, maio/jun. 1996.

\section{EUROPEAN COMMITTEE FOR}

STANDARDIZATION. EN 312: particleboard, specifications. Brussels, 2003.

GUIMARÃES JÚNIOR, J. B. Painéis de madeira de eucalipto: estudo de caso de espécies e procedências. 2008. 95 p. Dissertação (Mestrado em Engenharia Florestal) - Universidade Federal de Lavras, Lavras, 2008.

IWAKIRI, S. Painéis de madeira reconstituída. Curitiba: FUPEF, 2005. 274 p.

IWAKIRI, S.; CUNHA, A. B.; ALBUQUERQUE, C. E. C.; GORNIAK, E.; MENDES, L. M. Resíduos de serrarias na produção de painéis de madeira aglomerada de Eucalyptus. Scientia Agrária, Curitiba, v. 1, n. 2, p. 23-28, 2000.

MALONEY, T. M. Modern particleboard and dryprocess fiberboard manufacturing. 2 nd ed. San Francisco: M. Freeman, 1993. 689 p.

MENDES, R. F. Efeito do tratamento térmico sobre as propriedades de painéis OSB. 2011. 115 p. Dissertação (Mestrado em Engenharia Florestal) - Escola Superior de Agricultura "Luiz de Queiroz", Piracicaba, 2011.

MORAIS, S. A. L.; NASCIMENTO, E. A.; MELO, D. C. Análise da madeira de Pinus oocarpa: parte I, estudo dos constituintes macromoleculares e extrativos voláteis. Revista Árvore, Viçosa, v. 29, n. 3, p. 461-470, maio/ jun. 2005.

NAUMANN, R. B.; VITAL, B. R.; CARNEIRO, A. C. O.; DELLA LUCIA, R. M.; SILVA, J. de C.; CARVALHO, A. M. M. L.; COLLI, A. Propriedades de chapas fabricadas com partículas de madeira de Eucalyptus urophylla S. T. Blake e de Schizolobium amazonicum herb. Revista Árvore, Viçosa, v. 32, n. 6, p. 1143-1150, nov./dez. 2008.

\section{NORMEN FÜR HOLZFASERPLATEN}

SPANPLATTEN SPERRHOLZ. Testing of wood chipboards bending test, determination of bending strength: DIN 52362. Berlin, 1982.

QUEIROZ, S. C. S.; GOMIDE, J. L.; COLODETTE, J. L.; OLIVEIRA, R. C. Influência da densidade básica da

Cerne, Lavras, v. 20, n. 2, p. 329-336, abr./jun. 2014 madeira na qualidade da Polpa kraft de clones hibrídos de Eucalyptus grandis w. Hill ex Maiden x Eucalyptus urophylla s. T. Blake. Revista Árvore, Viçosa, v. 28, n. 6, p. 901-909, nov./dez. 2004.

PEDRAZZI, C.; HASELEIN, C. R.; SANTINI, E. J.; SCHNEIDER, P. R. Qualidade de chapas de partícula de madeira aglomerada fabricadas com resíduo de uma indústria de celulose. Ciência Florestal, Santa Maria, v. 16, n. 2, p. 201-212, 2006.

TRIANOSKI, R. Avaliação do potencial de espécies florestais alternativas de rápido crescimento para produção de painéis de madeira aglomerada. 2010. 260 p. Dissertação (Mestrado em Ciências Florestais) Universidade Federal do Paraná, Curitiba, 2010.

TRUGILHO, P. F.; LIMA, J. T.; MENDES, L. M. Influência da idade nas caracterís-ticas físico-mecânicas e anatômicas da madeira de Eucalyptus saligna. Cerne, Lavras, v. 2, n. 1, p. 97-111, jan./jun. 1996.

TSOUMIS, G. Science and technology of wood: structure, properties, utilization. New York: V. N. Reinhold, 1991. 494 p.

$\overline{\text { Recebido: } 26 \text { de junho de 2011; aceito: } 01 \text { de outubro de } 2013 .}$ 